\title{
Predicting the likelihood of postoperative seizure status based on mRNA sequencing in low-grade gliomas
}

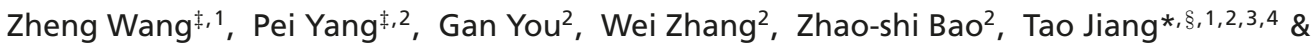 \\ Chuan-bao Zhang**, $\$, 1$ \\ ${ }^{1}$ Beijing Neurosurgical Institute, Capital Medical University, Beijing, PR China \\ ${ }^{2}$ Department of Neurosurgery, Beijing Tiantan Hospital, Capital Medical University, Beijing, PR China \\ ${ }^{3}$ China National Clinical Research Center for Neurological Diseases, Beijing, PR China \\ ${ }^{4}$ Center of Brain Tumor, Beijing Institute for Brain Disorders, Beijing, PR China \\ * Author for correspondence: Tel./Fax: +86 106709 8431; taojiang1964@163.com \\ ** Author for correspondence: Tel./Fax: +86 106709 8431; chuanbao123@139.com \\ $¥$ Authors contributed equally \\ $\S$ Co-senior authors
}

\begin{abstract}
Aim: No comprehensive and objective methods yet exist for predicting postoperative seizure. Patients \& methods: mRNA-seq data and corresponding postoperative seizure status of 109 low-grade glioma samples were obtained from Chinese Glioma Genome Atlas database and divided into two sets randomly. Logistic regression and receiver operating characteristic analysis with risk score method were used to develop a ten-gene prediction model. Results: Considering gene number and area under the curve of receiver operating characteristic, a ten-gene model was generated which showed an area under the curve of 0.9965 in training set. Patients with high-risk scores had higher probability of postoperative seizure compared with those with low-risk scores. Conclusion: This is the first prediction model for postoperative seizures in gliomas, integrating multiple genes. Clinical application may help patients with postoperative seizure control.
\end{abstract}

First draft submitted: 1 November 2017; Accepted for publication: 22 November 2017; Published online: 5 December 2017

Keywords: low-grade glioma $\bullet$ mRNA-seq $\bullet$ postoperative seizure $\bullet$ predicting model

Seizure is a common presenting symptom of primary brain tumors. Seizure incidence varies with tumor type, grade and location. Low-grade tumors are more epileptogenic than high-grade tumors [1]. Although most patients (65-90\%) with low-grade gliomas (LGGs) experience symptomatic seizures [2], many do not have seizures in spite of similar histology and tumor location. This indicates that the variability of seizure occurrence cannot be explained solely by peritumoral factors or histology, urging us to explore the genetic factors that may be associated with tumor-related seizure. The pathogenesis of tumor-related epilepsy seems to be different from that of idiopathic epilepsy, leading to distinct clinical characteristics and treatment [3].

Seizure, as one of the most common postoperative symptom in patients with LGGs [4-7], when uncontrolled, would badly affect patients' quality of life (QOL), causing cognitive deterioration and may result in significant morbidity [8-11]. Postoperatively uncontrolled seizures and subsequent use of anti-epileptic drugs (AEDs) would continuously affect QOL and cognitive function $[8,10]$. Although two-thirds of patients with LGGs would experience seizures after surgery [3], not all LGGs are associated with postoperative seizures, despite of similar histology and tumor location. Therefore, predicting postoperative seizure in patients with diffuse LGGs is valuable [12].

Driven by progress in next-generation sequencing, comprehensive interpretation of the tumor biology has been broadly deployed in various cancers [13]. Transcriptome sequencing, especially mRNA-seq, provides a lower cost, more precise insight into the complex transcriptome of the tumors on gene expression, etc. By combining the most advanced technology and the detailed follow-up of patients, we set out to predict the postoperative seizures status, which may help with seizure control postoperatively. 
Although several factors are known to influence the likelihood of postoperative seizures, including WHO grade, gross total resection and Ki67 expression level [3], to date, we fail to find any mRNA-seq-based prediction model for postoperative seizures in glioma. In this study, we developed a statistical model that can accurately predict the likelihood of postoperative seizures, which may help patients with postoperative seizure control.

\section{Patients \& methods}

\section{Patients \& samples}

Patients' data and samples were obtained from Chinese Glioma Genome Atlas (CGGA) dataset. Only patients with LGGs, and subsequently undergone surgical resection were enrolled into this study [3]. Studies on human material were approved by the institutional research ethics committee. Appropriate consent was obtained from human subjects for use of the material.

\section{mRNA-seq data}

MRNA-seq data were obtained from the CGGA database. Details on the procedures of sequencing have been described previously [14].

\section{Postoperative seizure outcome measures}

Data of seizure characteristics included date of seizure onset after operation and the use of AEDs. The primary outcome of seizure control was evaluated 12 months after surgery according to the Engel Classification Scheme of Seizures [15]. Positive samples were defined as those with improvement of postoperative seizures control (Engel class I, II and III), which the negative samples (Engel class IV) were not.

\section{Model construction}

One hundred and three samples with both mRNA-seq data and postoperative seizure follow-up data were enrolled into this study. All samples were randomly separated into two groups: a training set $(\mathrm{n}=52)$ and a validation set $(n=51)$. Logistic regression is a statistical approach to predict the presence of a certain status based on available variables, which has been successfully used for prediction and diagnosis in medicine [16,17]. In the training set, $\log 2$-transformed expression value of the remaining genes $(n=17,299)$ and the postoperative seizure status were applied to the logistic regression. The genes were then ranked according to the p-value of logistic regression. The risk score method was used to get a continuous risk prediction of postoperative seizure status. It was defined as sum of the product of top $\mathrm{n}$ gene expression values and corresponding $\beta$-values from logistic regression:

$$
\text { Risk score }=\sum_{i=1}^{n} \beta_{i} \cdot \text { Expression }_{\text {signature }(i)}
$$

To screen for genes with best prediction value, receiver operating characteristic (ROC) curve was obtained along with the area under the curve (AUC) of top n genes. Considering the number of genes and AUC, the top ten genes approached to a peak, which were used to build the prediction model. The risk score of every individual was calculated according to the formula above and the cutoff was defined as the 30th percentage (cutoff $=0.5538$ ) of the risk scores, which is similar with the proportion of postoperative seizure $(34 / 103)$. Those with higher risk scores were statistically defined as suffering from postoperative seizure, while the others were not.

\section{Model evaluation}

In the validation dataset, the risk scores based on the ten-gene model were calculated according to the formula above, with the same $\beta$-values and cutoff. With the same definition, those with higher risk scores were statistically considered as suffering from postoperative seizure, while the others were not. All the procedures were repeated in the whole dataset for further verification.

In the training set, differently expressed genes between positive and negative samples were evaluated by t-test, adjusted by Benjamini \& Hochberg false discovery rate.

All the statistical analyses were performed in $\mathrm{R}$ [18]. ROC curves were obtained by the pROC package compiled under $\mathrm{R}$ language[19] of $\mathrm{R}$ and heatmaps were obtained by the pheatmap package [20] of $\mathrm{R}$. 


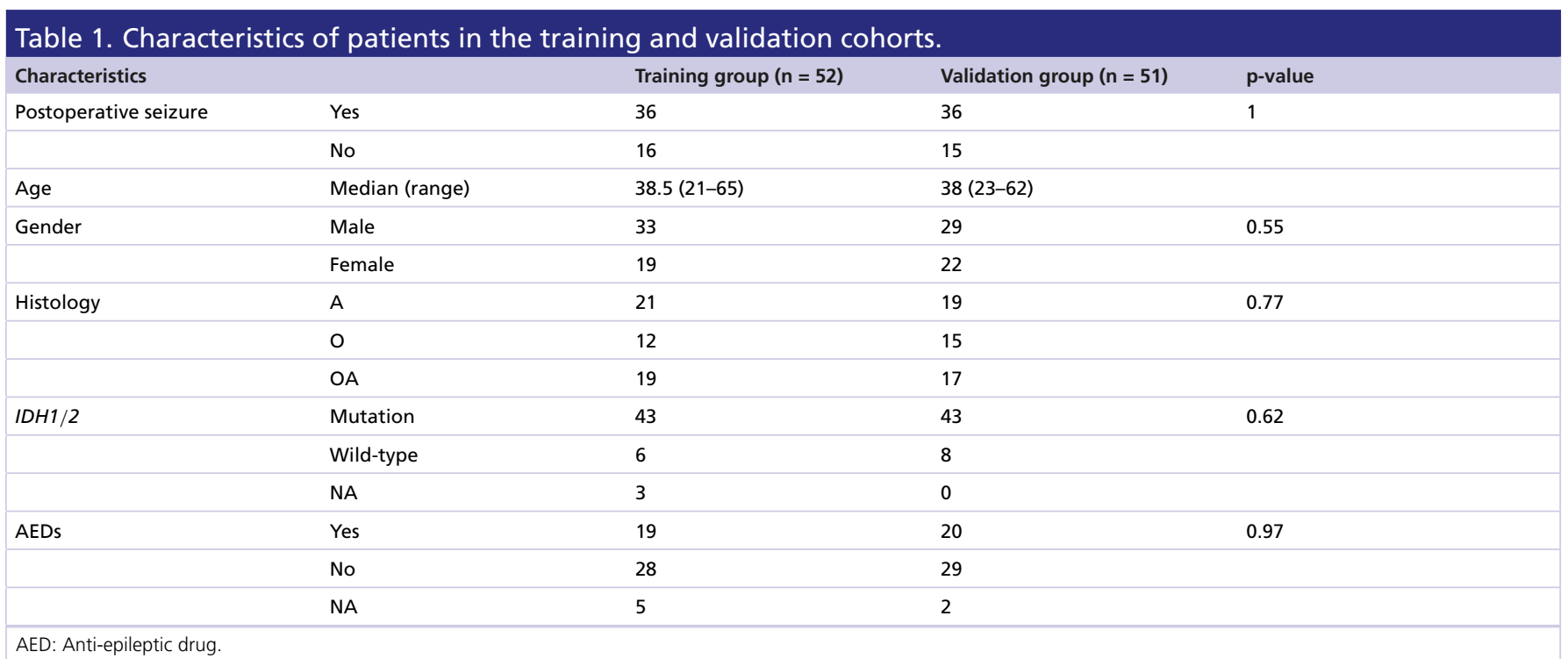

Table 2. Top ten genes significantly associated with postoperative seizure by logistic regression in the training set.

\begin{tabular}{|lll|}
\hline Gene symbol & $\boldsymbol{\beta}$ & $\boldsymbol{p}$-value \\
\hline PCDHB6 & 1.073014 & 0.003774 \\
\hline TOMM2OL & -2.48657 & 0.004703 \\
\hline SCRG1 & -1.19577 & 0.00573 \\
\hline GYPE & -0.87181 & 0.008233 \\
\hline ZDHHC20 & -0.84531 & 0.008319 \\
\hline TUBGCP5 & -0.9549 & 0.011906 \\
\hline ZNF839 & -0.78928 & 0.01234 \\
\hline CCL21 & 0.935245 & 0.013347 \\
\hline LOC100507632 & -0.86902 & 0.013451 \\
\hline SCNM1 & -0.84486 & 0.014029 \\
\hline
\end{tabular}

\section{Results}

\section{Characteristics of the samples}

Of these 103 patients, 52 were randomly divided into training group, and the other 51 were assigned into validation group. Between these two groups, there were no significant differences in incidence rate of postoperative seizure, age, gender, histological types, IDH1/2 mutation and use of AEDs (Table 1).

\section{Distinctive expression profile of the positive and negative samples}

Hierarchical clustering of the top 100 differently expressed genes indicated the distinctive expression profile of the two groups (Figure 1A). Hierarchical clustering of the correlation between every two samples was also performed (Figure 1B), which suggested a clear pattern; almost all the positive samples clustered together, and vice versa. The different expression profile laid the foundation of building a prediction model based on mRNA expression.

\section{Model construction in training set}

As mentioned in the 'Patients \& methods' section, the top ranked ten genes after logistic regression approached to a peak of the AUC (Figure 2A \& B), which indicated the best performance of the model. The top ten genes and the corresponding $\beta$-values were listed in Table 2 .

By applying the model in the training set, 52 samples were stratified into positive and negative groups (Figure 2C), which was in high accordance with the follow-ups. This model generated an AUC of 0.9965 (95\% CI: 0.9882, 1; computed with 2000 stratified bootstrap replicates), a sensitivity of $97.22 \%$ and a specificity of $93.75 \%$ in training set (Figure 2D). 


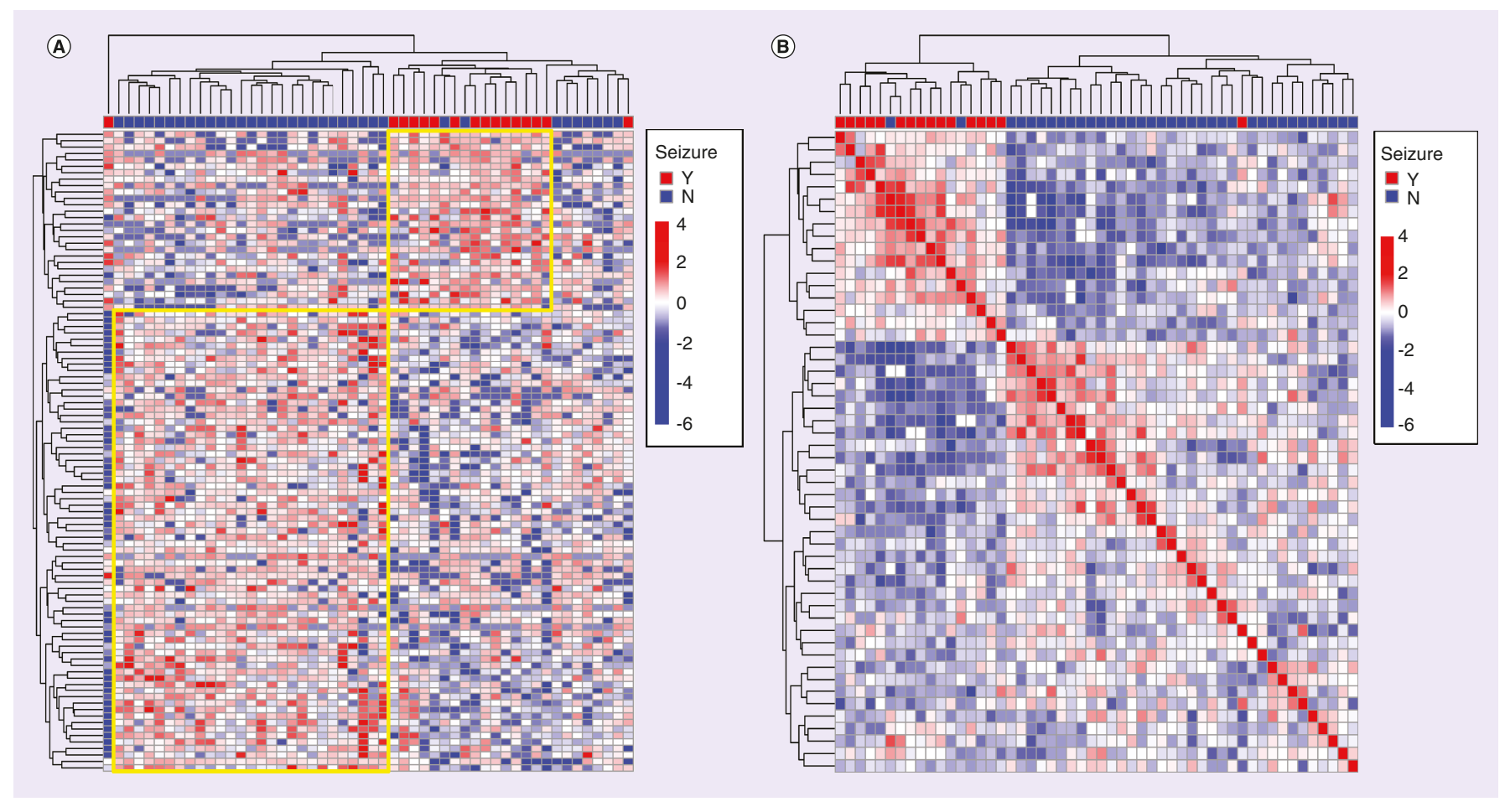

Figure 1. Patients with postoperative seizure showed distinct expression profiles. (A) Unsupervised clustering of the expression profiles of LGG patients from Chinese Glioma Genome Atlas database based on the top 100 differently expressed genes. The expression subtypes were correlated with postoperative seizure status. (B) Clustering of the correlations between every two samples. The correlation subtype showed tight association with postoperative seizure status.

LGG: Low-grade glioma; N: No; Y: Yes.

Model evaluation in the validation set

In the validation dataset, the same ten genes and $\beta$-values were applied to get the corresponding risk scores of the 51 samples. Similarly, 30 out of the 36 (sensitivity: 83.33\%) patients with postoperative seizures were predicted correctly by this model, while $11 / 15$ (specificity: $73.33 \%$ ) patients were predicted to be negative (Figure $3 \mathrm{~A}$ ). This model had an AUC of 0.8593 (95\% CI: 0.7572-0.9613; Figure 3B).

Taken together, of the 103 patients included in this study, this model generated a sensitivity of $90.28 \%$, a specificity of $83.87 \%$ (Figure 3C) and an AUC of 0.9391 (95\% CI: 0.8943-0.9838; Figure 3D). The high accuracy of this model highlighted the biological significance of the ten genes in the diagnosis and treatment of postoperative seizures.

\section{Discussion}

Recently, effective ways of predicting the risk of tumor-related seizures are still limited. Cerebral hemisphere LGGs are one of the most epileptogenic cerebral lesions while patients with LGGs usually survive for 5-10 years. Seizures will badly damage the QOL of these patients. Identification of predictors for postoperative seizures is essential and requires studies based on large series of patients over a long follow-up. Although several variables including clinical factors [1] as well as molecular biomarkers [3,21] are known to be associated with postoperative seizures, many cases may bear paradoxical seizure-related factors at the same time. For example, a 20-year-old patient with grade II oligodendroglioma located within functional areas, and bearing mutant IDH1 in the tumor, may suffer from postoperative seizures. But it is less likely obvious what to do with an IDH1 wild-type oligoastrocytoma in a 70-year-old patient without functional areas involvement. The predicting model based on mRNA-seq described here, utilizing advanced statistical modeling to produce a probability of postoperative seizures, should therefore be useful to clinicians, pathologists and researchers.

Recent studies on that topic revealed that postoperative seizure frequencies vary between studies, including one monocentric study of 508 patients [3], one multicenter study of 1509 patients [1] and a systematic literature review 
(A)

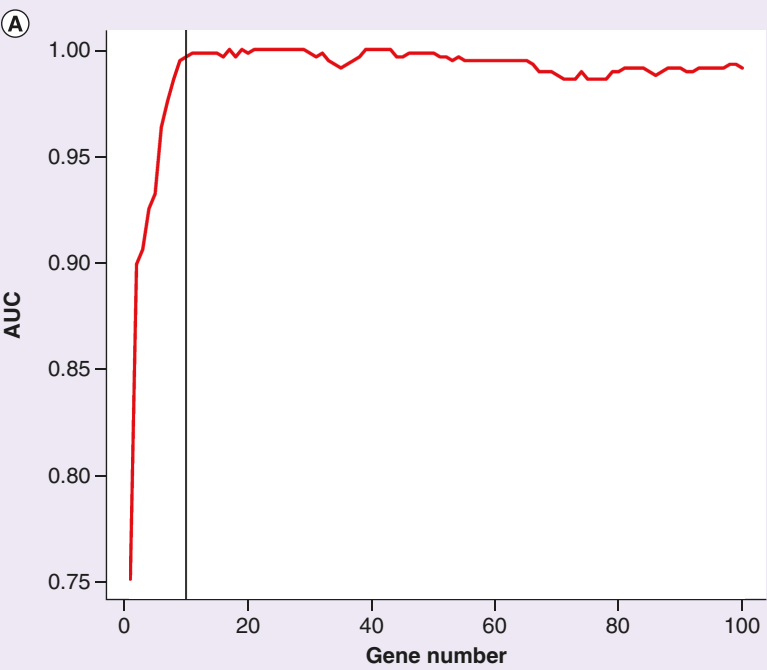

(C)

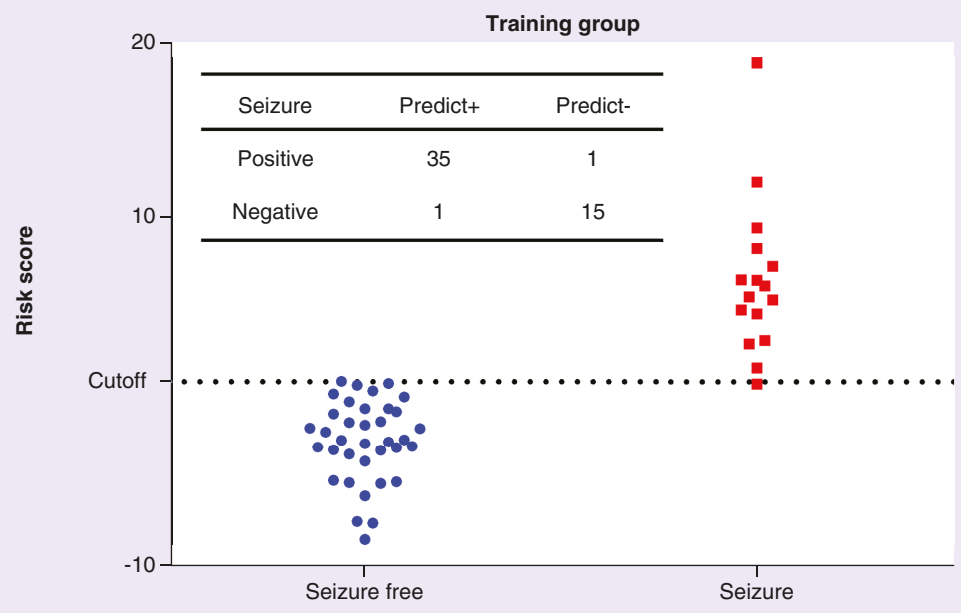

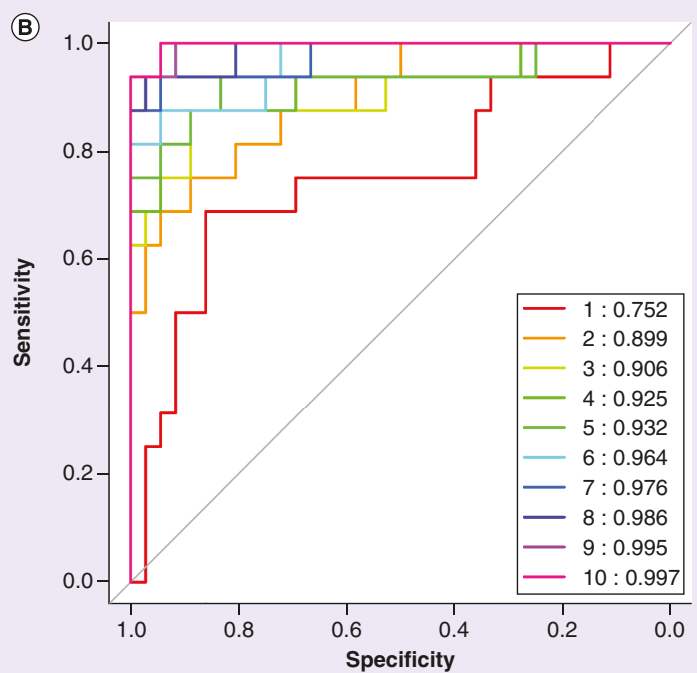

(D)

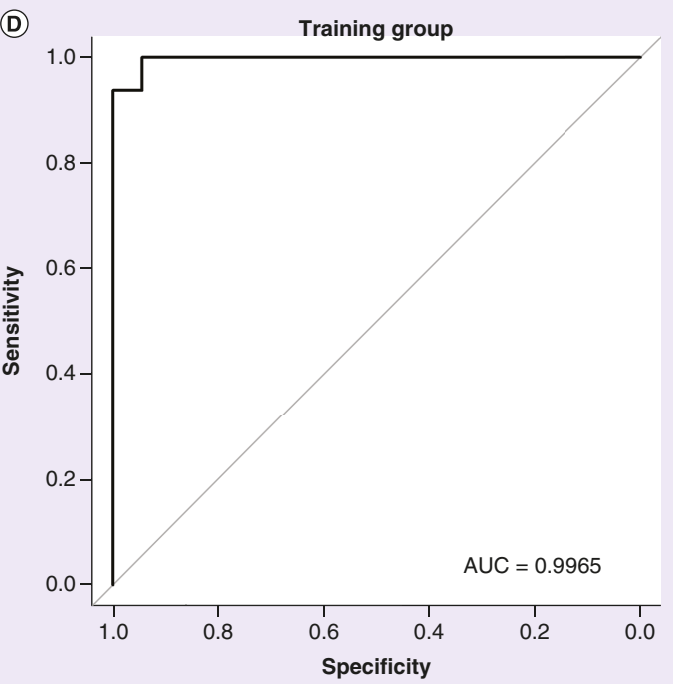

Figure 2. Construction of the prediction model in the training dataset $(\mathbf{n}=\mathbf{5 2})$. (A) Consecutive AUCs of the top (1-100) genes in prediction of postoperative seizure status. The top ten genes achieved the plateau of AUC. (B) The representative AUCs of the top (1-10) genes were shown in the ROC curves. (C) Patients in the training set were predicted to be seizure free or with seizure by applying the model. (D) The AUC of the final prediction model was assessed by the ROC curve.

AUC: Area under the curve; ROC: Receiver operating characteristic.

with meta-analysis, pooling 773 patients from 20 small-sized studies [22]. Though well conducted and interesting, the contribution of these studies is limited by their accuracy of the predictors to postoperative seizure.

In this study, by applying logistic regression and risk score method, we built a clinically applicable prediction model for postoperative seizures. Of the ten genes involved, $P C D H B G$ belongs to the protocadherin beta gene cluster, which most likely play a critical role in the establishment and function of specific cell-cell neural connections [23]. TOMM20L, the ligand of TOMM20, which localized at the outer mitochondrial membrane, is associated with metabolism [24]. SCRG1, is associated with neurodegenerative changes observed in transmissible spongiform encephalopathies [25]. SCNM1, sodium channel modifier 1, a zinc finger protein and a putative splice factor, is involved in the splicing of sodium channel transcripts [26]. Although none of these genes has been widely reported to be associated with postoperative seizures, they somehow show relationship with cell-cell interaction, cell metabolism or ion transportation, which are thought to be associated with seizures.

Although this model was developed from high-throughput mRNA-seq data in a large cohort of patients, it still need further validation in other datasets. Limited by the current shortage of sequencing data with long-term seizure follow-ups, the comprehensive evaluation of the model may be finished in the near future. 
(A)

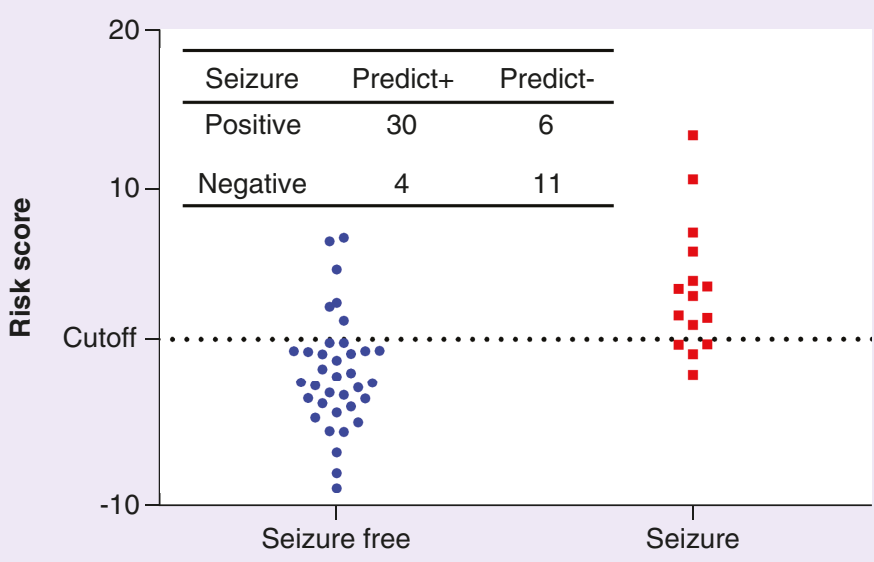

(C)

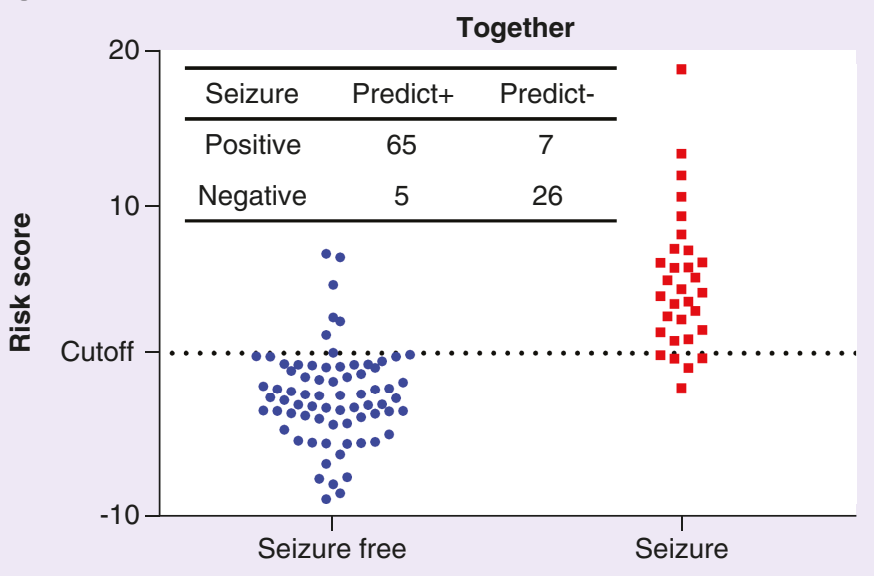

(B)

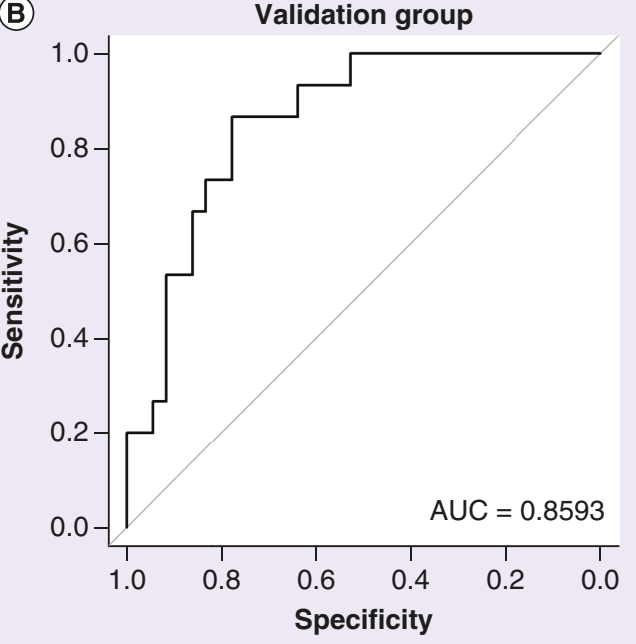

(D)

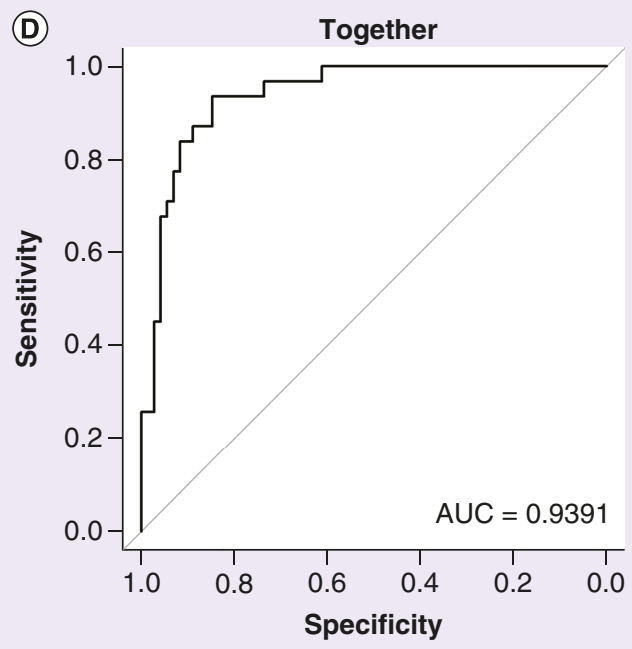

Figure 3. Validation of the prediction model in the validation dataset $(n=51)$ and the whole dataset $(n=103)$. (A) Patients in the validation set were predicted to be seizure free or with seizure by applying the model. (B) The AUC the model in validation dataset was evaluated by ROC curve. (C) The whole cohort of patients was predicted to be seizure free or with seizure by applying the model. (D) This model had a AUC of 0.9391 in the whole dataset.

AUC: Area under the curve; ROC: Receiver operating characteristic.

\section{Conclusion}

In summary, we established a prediction model in this study, which showed a high sensitivity and specificity in postoperative seizure prediction. This is the first seizure prediction model derived from mRNA-seq data. The application may help patients with postoperative seizure control.

\section{Summary points}

- This is the first molecular prediction model for postoperative seizures in gliomas, integrating multiple genes to generate a probability of postoperative seizures.

- Clinical application of this model may help patients with postoperative seizure control.

\section{Disclaimer}

This report was written by experts selected by the International League Against Epilepsy (ILAE) and was approved for publication by ILAE. Opinions expressed by the authors, however, do not necessarily represent the policy or position of ILAE. 
Financial \& competing interests disclosure

This study was supported by grants from The National Key Research and Development Plan (grant number: 2016YFC0902500), Beijing Science and Technology Plan (grant numbers: Z131100006113018 and Z141100000214009), Capital Medical Development Research Fund (grant number: 2016-1-1072) and National Natural Science Foundation of China (grant numbers: 81301112 and 81502606), Jiangsu Province thirteen-five-year science and education project for young people talent (QNRC2016189). The authors have no other relevant affiliations or financial involvement with any organization or entity with a financial interest in or financial conflict with the subject matter or materials discussed in the manuscript apart from those disclosed.

No writing assistance was utilized in the production of this manuscript.

\section{Ethical conduct of research}

The authors confirm that they have read the Journal's position on issues involved in ethical publication and affirm that this report is consistent with those guidelines.

\section{Open access}

This work is licensed under the Attribution-NonCommercial-NoDerivatives 4.0 Unported License. To view a copy of this license, visit http://creativecommons.org/licenses/by-nc-nd/4.0/

\section{References}

1 Pallud J, Audureau E, Blonski M et al. Epileptic seizures in diffuse low-grade gliomas in adults. Brain 137(Pt 2), 449-462 (2014).

2 Lee JW, Wen PY, Hurwitz S et al. Morphological characteristics of brain tumors causing seizures. Arch. Neurol. 67(3), 336-342 (2010).

3 You G, Sha ZY, Yan W et al. Seizure characteristics and outcomes in 508 Chinese adult patients undergoing primary resection of low-grade gliomas: a clinicopathological study. Neuro Oncol. 14(2), 230-241 (2012).

4 Chang EF, Potts MB, Keles GE et al. Seizure characteristics and control following resection in 332 patients with low-grade gliomas. J. Neurosurg. 108(2), 227-235 (2008).

5 Fried I, Kim JH, Spencer DD. Limbic and neocortical gliomas associated with intractable seizures: a distinct clinicopathological group. Neurosurgery 34(5), 815-823 (1994).

6 White JC, Liu CT, Mixter WJ. Focal epilepsy - a statistical study of its causes and the results of surgical treatment: epilepsy secondary to cerebral trauma and infection. N. Engl. J. Med. 239(1), 1-10 (1948).

7 Yang P, Wang Y, Peng X et al. Management and survival rates in patients with glioma in China (2004-2010): a retrospective study from a single-institution. J. Neurooncol. 113(2), 259-266 (2013).

8 Klein M, Engelberts NH, van der Ploeg HM et al. Epilepsy in low-grade gliomas: the impact on cognitive function and quality of life. Ann. Neurol. 54(4), 514-520 (2003).

9 Sheth RD. Adolescent issues in epilepsy. J. Child Neurol. 17(Suppl. 2), 2S23-2S27 (2002).

10 Taphoorn MJ, Klein M. Cognitive deficits in adult patients with brain tumours. Lancet Neurol. 3(3), 159-168 (2004).

11 Zaatreh MM, Firlik KS, Spencer DD, Spencer SS. Temporal lobe tumoral epilepsy: characteristics and predictors of surgical outcome. Neurology 61(5), 636-641 (2003).

12 Chang EF, Smith JS, Chang SM et al. Preoperative prognostic classification system for hemispheric low-grade gliomas in adults. J. Neurosurg. 109(5), 817-824 (2008).

13 Doroshow JH, Kummar S. Translational research in oncology - 10 years of progress and future prospects. Nat. Rev. Clin. Oncol. 11(11), 649-662 (2014).

14 Bao ZS, Chen HM, Yang MY et al. RNA-seq of 272 gliomas revealed a novel, recurrent PTPRZ1-MET fusion transcript in secondary glioblastomas. Genome Res. 24(11), 1765-1773 (2014).

15 Engel J Jr, International League Against Epilepsy (ILAE). A proposed diagnostic scheme for people with epileptic seizures and with epilepsy: report of the ILAE Task Force on Classification and Terminology. Epilepsia 42(6), 796-803 (2001).

16 Bagley SC, White H, Golomb BA. Logistic regression in the medical literature: standards for use and reporting, with particular attention to one medical domain. J. Clin. Epidemiol. 54(10), 979-985 (2001).

17 Gareen IF, Gatsonis C. Primer on multiple regression models for diagnostic imaging research. Radiology 229(2), 305-310 (2003).

18 Team RC. R: A Language and Environment for Statistical Computing. R Foundation for Statistical Computing, Vienna, Austria (2013).

19 Robin X, Turck N, Hainard A et al. pROC: an open-source package for R and S+ to analyze and compare ROC curves. BMC Bioinformatics 12, 77 (2011).

20 Kolde R. Pheatmap: pretty heatmaps (2013). https://cran.r-project.org/web/packages/pheatmap/index.html

21 Huang L, You G, Jiang T, Li G, Li S, Wang Z. Correlation between tumor-related seizures and molecular genetic profile in 103 Chinese patients with low-grade gliomas: a preliminary study. J. Neurol. Sci. 302(1-2), 63-67 (2011). 
22 Englot DJ, Berger MS, Barbaro NM, Chang EF. Predictors of seizure freedom after resection of supratentorial low-grade gliomas. A review. J. Neurosurg. 115(2), 240-244 (2011).

23 Yagi T, Takeichi M. Cadherin superfamily genes: functions, genomic organization, and neurologic diversity. Genes Dev. 14(10), 1169-1180 (2000).

24 Hernandez JM, Giner P, Hernandez-Yago J. Gene structure of the human mitochondrial outer membrane receptor Tom20 and evolutionary study of its family of processed pseudogenes. Gene 239(2), 283-291 (1999).

25 Dron M, Dandoy-Dron F, Guillo F et al. Characterization of the human analogue of a Scrapie-responsive gene. J. Biol. Chem. 273(29), 18015-18018 (1998).

26 Buchner DA, Trudeau M, Meisler MH. SCNM1, a putative RNA splicing factor that modifies disease severity in mice. Science 301(5635), 967-969 (2003). 